\section{Tamás Kiss Xavier Darzacq}

ADRESSE

T. Kiss, X. Darzacq : Laboratoire de biologie moléculaire eucaryote du Cnrs, 118, route

\title{
Plus d'un siècle après sa découverte, un nouveau regard sur le nucléole
}

Le nucléole a été la première structure subnucléaire décrite par les microscopistes, et a longtemps été considéré comme un organite hautement spécialisé dans la biosynthèse des ribosomes. Plus d'un siècle après sa découverte, des études récentes ont montré que le nucléole est également utilisé par la cellule pour accomplir des fonctions cellulaires très diverses. En effet, il est impliqué dans la biogenèse de nombreux ARN cellulaires transcrits dans le noyau et, de manière surprenante, il joue un rôle important dans des mécanismes tels que le contrôle du cycle cellulaire, la modulation de l'activité de p53 et le contrôle de la méiose. Le nucléole ne peut donc plus être considéré uniquement comme le lieu de synthèse des ribosomes. Le «nucléole plurifonctionnel » reste à ce jour un organite peu connu, qui réserve certainement encore bien des surprises.

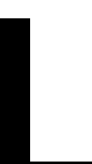

e nucléole est un organite subnucléaire, décrit dès le $\mathrm{XIX}$ siècle par les microscopistes grâce à sa structure lense en protéines et en ARN [1, 2]. II est présent dans le noyau des cellules interphasiques, et constitue une structure extrêmement dynamique, capable de se dissocier au moment de l'entrée en mitose, puis de se reformer au cours de la télophase.

Le nucléole s'assemble autour de locus chromosomiques contenant des centaines de gènes d'ARN ribosomique ( $A R N r)$, répétés en tandem, dont la transcription est essentielle pour sa formation. Le transcrit naissant de l'ARN pré-ribosomique (préARN r) est certainement I'élément qui nuclée l'assemblage de facteurs protéiques. Ceux-ci recrutent les éléments de la machinerie de synthèse des ribosomes, formant ainsi la structure nucléolaire.

Au cours de la mitose, les facteurs de la machinerie de maturation des ribosomes forment des structures pré-nucléolaires en $s^{\prime}$ associant aux transcrits de pré-ARNr pré-existants. $L$ 'existence de ces structures prénucléolaires au cours de la mitose 
démontre que les composants du nucléole peuvent s'assembler sans que la machinerie de transcription des gènes des $A R N$ ribosomiques soit active.

Dans les années 1960, le nucléole était considéré comme "l'usine à ribosomes» de la cellule. Le nucléole d'une cellule humaine $\mathrm{HeL}$ a en phase de croissance exponentielle synthétise environ 150 ribosomes à la seconde, qui doivent être exportés vers le cytoplasme à travers les pores nucléaires. Ce rythme de synthèse implique l'importation d'environ 12000 protéines ribosomiques à la seconde, du cytoplasme vers le nucléole, à travers ces mêmes pores nucléaires. Des expériences de photo-atténuation ont montré qu'outre les composants des ribosomes, de nombreux facteurs nucléolaires transitent rapidement entre le nucléole et le nucléoplasme qui l'entoure [3], suggérant l'existence d'une interconnexion fonctionnelle importante entre ces deux compartiments.

Les mécanismes de la synthèse nucléolaire des ribosomes, comprenant la transcription des pré-ARN ribosomiques, la formation d'ARN ribosomiques mûrs par clivages successifs des pré-ARN r et l'assemblage de sous-unités ribosomiques à partir des ARNr, ont récemment été résumés dans d'excellentes revues [4].

Dans cette synthèse, nous avons choisi de nous concentrer sur les découvertes récentes de nouvelles fonctions du nucléole. Nous verrons que ces découvertes ne nous permettent plus de considérer le nucléole uniquement comme un organite hautement spécialisé dans la synthèse des ribosomes.

\section{Modifications post- transcriptionnelles de différentes classes d'ARN cellulaires par des petits ARN nucléolaires (snoARN)}

A ce jour, les étudiants en deuxième cycle de biologie se représentent I'ARN comme un polymère d'uridine, cytidine, adénine et guanosine. Cette vue, bien que plus ou moins acceptable pour un ARN messager, est totalement erronée lorsque I'on considère les ARN cellulaires stables.
Les ARN de transfert ( $A R N t)$, ribosomiques (ARNr), et les petits ARN nucléaires ( SnARN), contiennent un grand nombre de nucléotides «irréguliers», issus de modifications posttranscriptionnelles covalentes des quatre bases classiques [5]. Six à $8 \%$ des ribo-nucléotides présents dans les cellules de vertébrés sont modifiés, reflétant l'énorme effort moléculaire que ces cellules concèdent à la synthèse de ces modifications.

Dans les ARNt, les nucléotides modifiés constituent des déterminants importants pour la spécificité et l'efficacité de la réaction d'aminoacylation, ainsi que pour la reconnaissance correcte des codons (pour revue, voir [6]). Chez les ARNr et les snARN, les nucléotides modifiés sont concentrés dans des régions fonctionnellement importantes et présentent une forte conservation phylogénétique, indiquant qu'ils jouent un rôle fondamental dans l'activité de ces ARN.

Bien qu'une centaine de modifications aient été répertoriées à ce jour, les méthylations du groupement hydroxyle en 2' du ribose, ainsi que les conversions d'uridine en pseudo- uridine, sont les plus rencontrées (figure 1). Les propriétés physico-chimiques des résidus méthylés en 2 '-O ribose et des pseudo-uridines, par rapport aux nucléotides dont ils dérivent, sont fondamentalement modifiées.

Une pseudo-uridine présente un groupement imine libre en position 1 (disponible pour engager une liaison hydrogène), qui n'est pas disponible dans l'uridine. Cette caractéristique confère à ces deux nucléotides des capacités d'interactions différentes avec leur environnement. La pseudo-uridine fut longtemps considérée comme le "cinquième ribonucléotide» des ARN cellulaires [7].

Pour les nucléotides méthylés en position 2'-0 -ribose, la capacité d'établir une liaison avec un hydrogène du groupement 2'-O-hydroxyle hydrophile est diminuée par le groupement méthyle hydrophobe.

Bien que la présence de pseudo-uridines, ainsi que de ribo-nucléotides méthylés en 2'-0-ribose, ait été décrite pour les ARN ribosomiques et pour d'autres ARN cellulaires, les bases moléculaires de ces modifications, dont la nature et la position

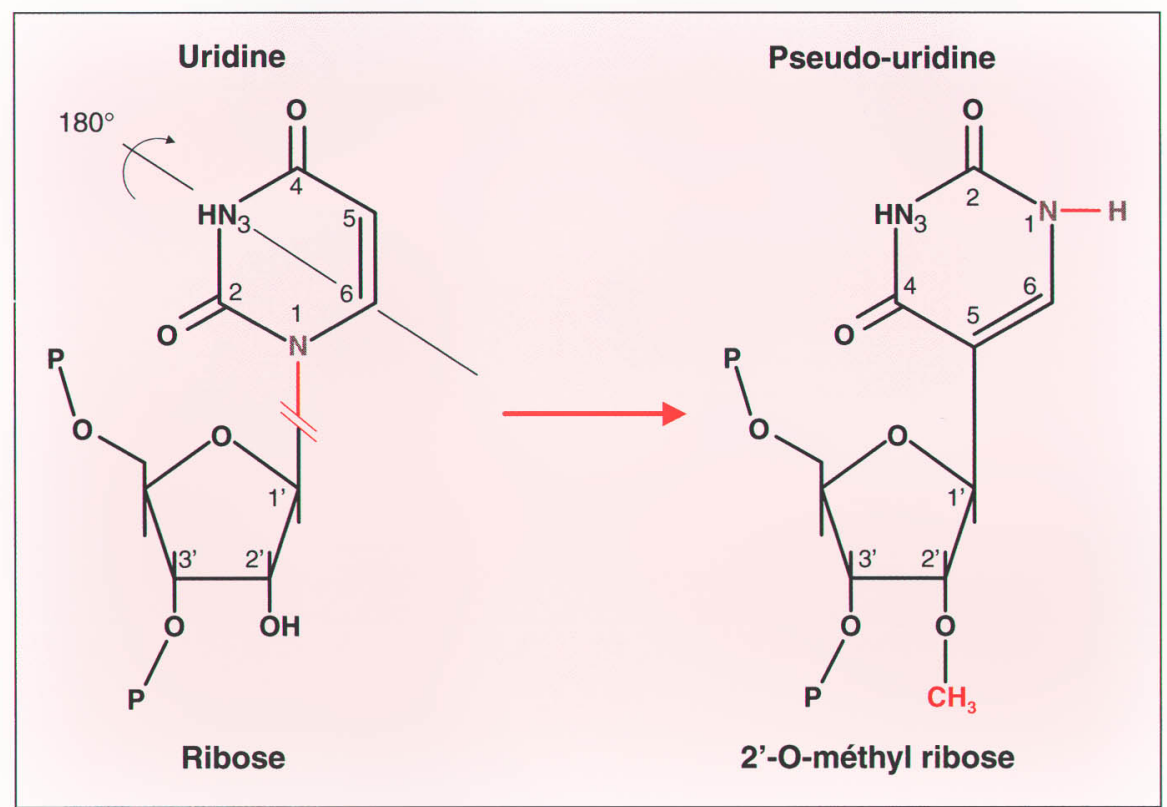

Figure 1. Synthèse de nucléotides méthylés en position 2'-O du ribose et de pseudo-uridines. La pseudo-uridine est issue d'une rotation de $180^{\circ}$ de la base d'une uridine. La pseudo-uridine est la base modifiée retrouvée la plus fréquemment dans la cellule, elle fut longtemps considérée comme le «cinquième nucléotide» de l'ARN [7]. La méthylation des quatre nucléotides en position 2'-O sur le ribose (groupement en rouge), constitue la modification post-transcriptionnelle la plus fréquente dans les ARN cellulaires. 
sont très spécifiques, sont restées énigmatiques pendant presque un demi-siècle.

\section{Il existe un grand nombre de petits ARN nucléolaires (snoAR N)}

Les nucléoles des cellules eucaryotes contiennent des centaines d'ARN métaboliquement stables, longs de 60 à 250 nucléotides, que l'on appelle les petits ARN nucléolaires ( SnoARN). Les snoARN forment une ribonucléoparticule (snoRNP) en $s^{\prime}$ associant à des protéines. Les snoARN peuvent être classés en deux sous-familles, définies par des séquences conservées et des éléments structuraux communs que nous détaillerons plus loin $[8,9]$. Bien que quelques snoARN soient impliqués dans la maturation nucléolytique des ARNr (pour revue, voir $[4,10]$ ). II est récemment devenu évident que la plupart des snoARN fonctionnent comme des guides dans les modifications post-transcriptionnelles des ARN r, des SnARN et certainement d'autres ARN cellulaires.

\section{Les snoAR N à boîtes $C$ et $D$ dirigent les méthylations en 2'-0 -ribose des ARN r}

L'une des deux familles majeures de SnOARN se caractérise par la présence d'une boîte $C$ phylogénétiquement conservée, dont la séquence consensus RUGAUGA est située à l'extrémité 5 ' de l'ARN, et d'une deuxième boîte $D$ (CUGA) située en 3' de I'ARN. Les extrémités du présnoARN s'apparient souvent entre elles pour former une courte hélice terminale. D'autres copies imparfaites des boîtes $C$ et $D$, appelées $C^{\prime}$ et $D^{\prime}$, sont souvent retrouvées dans la partie centrale de I'ARN. La plupart des ARN à boîtes $C$ et $D$ sont responsables de méthylations en position 2'0 -ribose des ARN ribosomiques $18 \mathrm{~S}$, $5.8 \mathrm{~S}$ et $28 \mathrm{~S}$. Ces SnoARN, guides de méthylation, portent de longues séquences (10-21 nucléotides), permettant un appariement parfait avec des séquences des ARNr. L'hélice d'ARN ainsi formée entre le snoARN et l'ARNr, place la boîte $D$ ou $D^{\prime}$ à exactement 5 nucléotides de la base de l'ARN $r$ qui sera méthylée en position 2'-O-ribose (figure 2A) [11, 12].

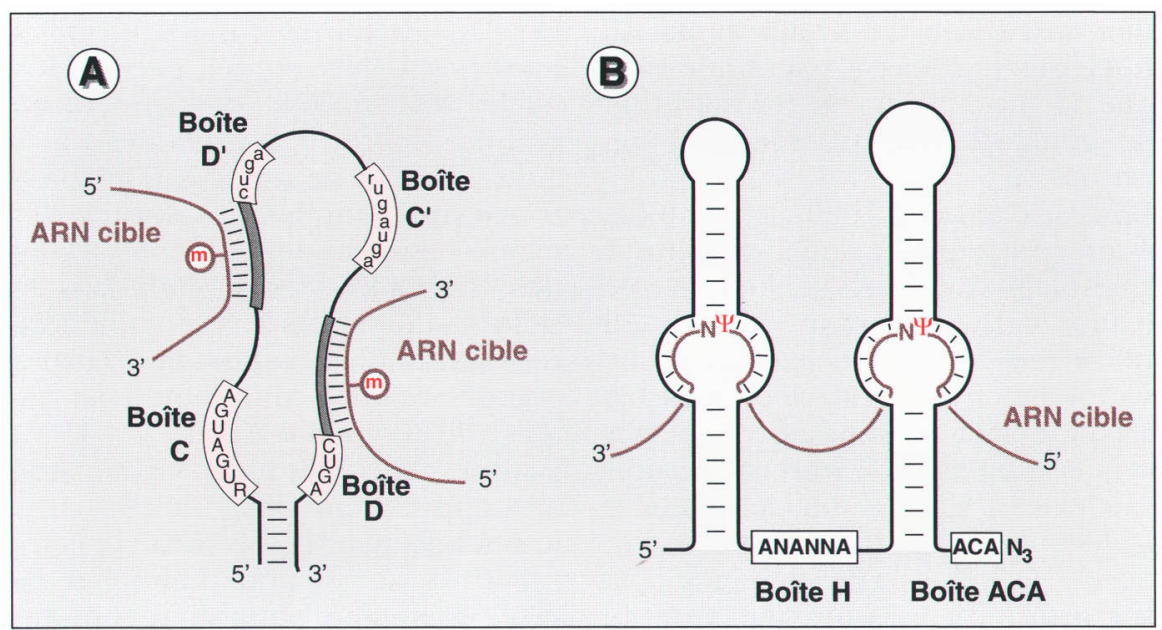

Figure 2. La plupart des petits ARN nucléolaires ont des fonctions de guides dans les modifications post-transcriptionnelles des ARN ribosomiques et des petits ARN nucléaires. A. Les snoARN à boîtes $C$ et $D$ sélectionnent les sites de méthylation en position 2'-O : Les snoARN, guides de méthylation en position 2'-O du ribose, contiennent un, ou quelquefois deux éléments «antisens », capables de former une double hélice d'ARN parfaite avec I'ARN cible. Cette interaction place la boîte D ou D' du snoARN à une distance de cinq bases de la position qui sera méthylée. B. Sélection des uridines sélectionnées pour être pseudo-uridilées par les snoARN à boîte H/ACA: Le snoARN forme un pseudo-nœud complexe avec son ARN cible (ARNr ou snARN). Dans cette structure, I'uridine substrat ( $\Psi)$, occupe invariablement la même position à la base de l'hélice qui ferme la boucle de reconnaissance du snoARN.

qui, en s'appariant à l'ARN r, permet la formation transitoire d'un consensus de séquence et de structure, qui apporte l'information concernant le positionnement du nucléotide à modifier. Dans cette structure snoARN/ ARN cible, les boîtes C/ D et $C^{\prime} / D^{\prime}$ contiennent l'information nécessaire à la fixation des facteurs protéiques de la snoRNP $[13,14]$. En 1993, une mutation ponctuelle portant sur la fibrilarine, l'une des protéines associées aux ARN à boîtes $C$ et $D$, fut caractérisée chez la levure. Chez ce mutant, la présence de bases méthylées sur le groupement 2 ' hydroxyle du ribose des ARN ribosomiques était abolie[15]. La découverte de l'implication des snoARN à boîtes $C$ et $D$ dans les méthylations en position 2'-O-ribose des ARN r fut par la suite I'un des premiers éléments de compréhension de ce phénotype [11]. Enfin, récemment, une signature de méthyltransférase a été mise en évidence dans la fibrilarine [16]. La mutation de la fibrilarine affectant les méthylations des ARN r porte en fait sur un acide aminé essentiel du domaine supposé de liai- son à la S-adénosine méthionine. La fibrilarine est ainsi un bon candidat pour accomplir la fonction enzymatique des snoRN $P$ à boîtes $C$ et $D$.

\section{Les SnoAR $N$ à boîtes $\mathrm{H}$ et ACA dirigent la formation de pseudo-uridines des AR Nr}

L'autre famille majeure de snoARN est caractérisée par une structure secondaire conservée au cours de l'évolution, consistant en deux tigesboucles connectées suivies par des régions simple brin (figure $2 B$ ). Les régions simple brin centrales et terminales portent respectivement les boîtes conservées $H$ (de séquence consensus ANANNA) et ACA (séquence consensus ACA) $[8,17]$. La plupart des snoARN à boîte H / ACA ont un rôle de guide dans la sélection des uridines des ARNr, qui sont converties en pseudo-uridines. Deux courtes séquences, s'appariant à l'ARNr de part et d'autre de l'uridine à modifier, sont responsables de la spécificité de ces ARN. Ces séquences complémentaires sont localisées dans une bulle interne de 
la tige $5^{\prime}$ ou de la tige $3^{\prime}$ du snoARN (voir figure 1B). L'appariement SnOARN-ARNr laisse libre l'uridine qui sera modifiée, permettant ainsi la rotation de la base au cours de la réaction d'isomérisation [17]. Le site de pseudo-uridylation est toujours placé en amont de la boîte $H$, ou de la boîte $A C A$, à une distance pouvant varier de 14 à 16 nucléotides par rapport à la pseudo-uridine [17, 18]. L'enzyme responsable de la réaction d'isomérisation, ici une pseudo-uridine synthase, est la protéine Nap57p (son homologue chez la levure est appelée $\mathrm{Cbf5)}$. Cette protéine est associée à tous les ARN présentant un motif H/ ACA $[19,20]$. Une mutation située dans la séquence codant pour Nap57 est responsable d'une maladie génétique, dyskeratosis congénita, dont nous discuterons plus loin.

\section{Les snoARN modifient aussi les snARN de la machinerie d'épissage des ARN pré-messagers}

Les petits SnARN U $1, \cup 2, \cup 4$, U 5 et U 6 sont parmi les composants clés d'un complexe de maturation des ARNm, le splicéosome, qui a pour rôle d'éliminer les introns non codants des pré-ARNm au cours du processus d'épissage. Chez les eucaryotes supérieurs, les cinq petits ARN majeurs du splicéosome contiennent à eux seuls 24 pseudo-uridines et 30 nucléotides 2 ' -0 -méthylés. Les facteurs responsables des modifications des huit nucléotides 2'-0-méthylés et des trois uridines converties en pseudo-uridines du SnARN U 6 ont été identifiés récemment dans le nucléole [21]. La synthèse d'au moins trois des nucléotides méthylés de $U 6$ est guidée par des petits ARN nucléolaires à boîtes $C$ et $D[22,21]$. Un étrange petit ARN hybride comportant les boîtes $C$ et $D$, ainsi que les boîtes $H$ et $A C A$, a été récemment identifié; il est à la fois responsable d'une méthylation en 2'-0-ribose, et d'une pseudo-uridylation sur I'ARN U 5 [23]. Notre laboratoire a très récemment identifié trois nouveaux guides dirigeant des modifications dans les SnARN U $1, \cup 2$ et $U 4$, ce qui suggère que les snoARN seraient impliqués dans les modifications posttranscriptionnelles de l'ensemble des SnARN de la machinerie d'épissage.
Afin que des snoARN présents dans le nucléole puissent modifier des snARN présents dans le nucléoplasme, il est nécessaire que l'un de ces deux partenaires puisse transiter dans le compartiment de l'autre. Ainsi, Iorsqu'il est injecté dans le nucléoplasme, le snARN U 6 peut être détecté transitoirement dans le nucléole d'ovocytes de xénope [24]. Jusqu'ici, aucune preuve expérimentale permettant d'affirmer que les SIARN U1, U 2, U 4 ou U 5 puissent eux aussi transiter par le nucléole n'a été apportée.

Deux snoARN à boîtes $C$ et $D$ récemment identifiés ne présentent aucune complémentarité ni avec l'ARN ribosomique, ni avec les snARN ou aucun autre petit ARN connu, ce qui laisse penser que les SnOARN pourraient modifier d'autres ARN encore inconnus [25]. De manière encore plus surprenante, trois autres petits ARN présentant une structure de guide de méthylation en position 2'-0-ribose et un guide potentiel de pseudo-uridylation, sans aucune séquence cible connue, sont exprimés spécifiquement dans le cerveau [26]. Ces résultats laissent présager que des snoARN pourraient avoir un rôle dans des modifications d'ARN spécifiques de tissus.

\section{Nucléole et biogenèse de petites riboparticules}

Différentes observations suggèrent qu'outre la biogenèse des ribosomes et des SnRNP, le nucléole pourrait jouer un rôle dans la maturation, I'assemblage et l'adressage intracellulaire de plusieurs autres complexes ribonucléoprotéiques (revue dans [27]).

\section{Particule de reconnaissance du signal}

La particule de reconnaissance du signal (SRP) est un complexe ribonucléoprotéique qui permet l'ancrage du ribosome à la membrane du réticulum endoplasmique, lorsque la traduction d'une protéine, portant un signal d'adressage membranaire ou sécrétoire, est déclenchée. II dirige ainsi la protéine en cours de synthèse vers la voie de l'exocytose. Lorsque de I'ARN SRP synthétisé in vitro est injecté dans le nucléoplasme, il migre très rapidement dans le nucléole avant d'être retrouvé dans le cytoplasme. Trois des cinq protéines composant la particule SRP mature ont été également détectées dans le nucléole, ce qui a permis de suggérer que l'assemblage de la ribonucléoparticule SRP s'y produit [28].

\section{Maturation des ARNt (RNase P)}

La maturation endonucléolytique de I'extrémité 5' des ARNt est catalysée par un complexe ribonucléoprotéique, la RN ase $P$. Cette protéine est localisée principalement dans le nucléoplasme, mais elle peut également être détectée dans le nucléole. Selon des résultats récents, la fraction nucléolaire de la RNase $P$ est responsable de la maturation des extrémités $5^{\prime}$ de certains ARNt, qui transitent par le nucléole au cours de leur maturation [29].

\section{Implication de la RNase MRP}

Le nucléole contient une autre ribonucléoparticule, la RN ase MRP. Cette endoribonucléase est impliquée dans la maturation nucléolytique des ARN ribosomiques. Cette enzyme est nécessaire à la viabilité cellulaire, mais son activité dans la maturation des ARN ribosomiques peut être abolie sans que la cellule en souffre, ce qui indique que la RN ase MRP est nécessaire à la maturation d'un ARN essentiel qui n'a pas encore été identifié.

\section{La télomérase}

La télomérase est un autre complexe enzymatique ribonucléoprotéique, responsable de la synthèse des ADN télomériques répétés à l'extrémité des chromosomes. L'une des protéines du complexe possède l'activité de transcriptase réverse, alors que la composante ARN de la particule joue ici le rôle de matrice pour cette synthèse d'ADN. De manière surprenante, la région terminale $3^{\prime}$ de I'ARN de la télomérase peut se replier suivant le consensus structural des snoARN à boîte H/ACA du nucléole. Le complexe riboprotéique de la télomérase contient les protéines connues pour s'associer aux snoARN à boîte H/ACA, et une fraction de la télomérase est retrouvée 
dans le nucléole $[30,31]$. Le domaine H/ACA de la télomérase pourrait dans ce cas permettre de stabiliser l'extrémité 3' de l'ARN, grâce à son association aux facteurs protéiques des snoARN à boîte H/ ACA dans le nucléole.

Le diskeratosis congénita (DKC), maladie génétique liée au chromosome $X$ évoquée plus haut, est causée par une mutation dans la séquence codant pour la Nap57, protéine spécifique des SnOARN à boîtes $\mathrm{H}$ et ACA, aussi appelée diskérine. Dans les extraits de cellules de patients atteints de DKC, le taux d'ARN télomérase est inférieur à la normale. II existe également une diminution de I'activité télomérase, aboutissant à un raccourcissement des télomères [32], ce qui explique le phénotype d'instabilité chromosomique observé précédemment dans des cultures de cellules provenant de patients atteints de DKC.

L'ensemble de ces observations démontre que le nucléole est un organite «plurifonctionnel», qui contribue à la biogenèse d'une grande diversité de complexes contenant des ARN, eux-mêmes impliqués dans un grand nombre de fonctions cellulaires.

\section{Nucléole et contrôle du cycle cellulaire}

La compartimentation de la cellule joue un rôle important dans différents processus cellulaires. Par exemple, la transcription de certains facteurs du cycle cellulaire est sous le contrôle d'activateurs de transcription, qui sont fréquemment séquestrés dans le cytoplasme. L'activation transcriptionnelle de ces facteurs est alors contrôlée par leur import dans le noyau par une machinerie d'import finement contrôlée en réponse à un stimulus défini. II a été démontré récemment que la cellule utilise le nucléole pour séquestrer des protéines contrôlant des étapes essentielles du cycle cellulaire, les empêchant ainsi d'exercer une activité sur leurs cibles présentes dans le nucléoplasme. Cette découverte dévoile un aspect inattendu des fonctions du nucléole dans le contrôle du cycle cellulaire. Nous allons la développer à travers trois exemples qui révélent I'extrême diversité de ces mécanismes.

\section{Sortie de mitose}

L'entrée de la cellule en mitose, ainsi que le déroulement correct de cette dernière, sont contrôlés par des kinases dépendantes des cyclines (CDK). En fin de mitose, les kinases sont inactivées par la dégradation protéolytique dépendante de I'APC (anaphase promoter complex) de leur cycline régulatrice. L'élément clé dans l'inactivation des kinases mitotiques est la phosphatase Cdc14, qui a pour cible $C$ dh 1 , un activateur essentiel du complexe APC [33, 34]. Des expériences d'immunolocalisation ont montré qu'au cours de la mitose, Cdc14 est maintenue dans le nucléole afin d'éviter que l'anaphase ne démarre prématurément. En fin de mitose, Cdc 14 transite du nucléole vers le nucléoplasme, où elle pourra déphosphoryler la protéine Cdh 1 (figure 3).

Cdc 14 est séquestrée dans le nucléole via son association avec Net1, une protéine nucléolaire qui interagit directement avec I'ADN ribosomique [35, 36]. Lorsque le gène de Netl est inactivé, Cdc14 est délocalisé dans le nucléoplasme ainsi que dans le cytoplasme tout au long du cycle cellulaire. Les cellules ainsi dépourvues de la protéine nucléolaire Netl sortent de mitose prématurément avant la fin de la ségrégation du matériel génétique.

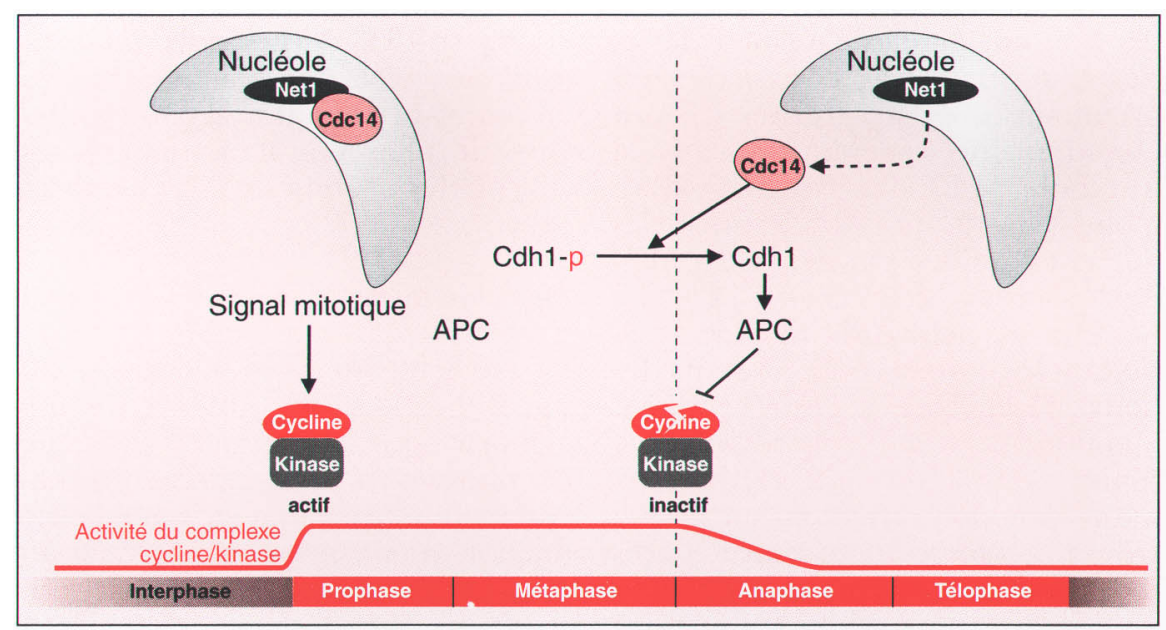

Figure 3. La protéine nucléolaire Net1 séquestre Cdc14 dans le nucléole pour maintenir la mitose. En début d'anaphase, Cdc14 est libérée du nucléole, elle peut alors déphosphoryler la protéine Cdh1, qui vient activer le complexe APC. Cette étape entraîne l'inactivation des CDK mitotiques par la dégradation protéolytique de leur cycline, permettant à la cellule de sortir de mitose.

\section{Modulation du taux d'expression de p53}

La protéine p53 est un suppresseur de tumeurs très important. En réponse aux dommages de I'ADN, ou à l'expression de certains oncogènes (Ras, M yc, E1A), p53 est stabilisée transitoirement dans le nucléoplasme, où elle induit la transcription de gènes responsables de l'arrêt du cycle cellulaire ou de l'entrée en apoptose. La concentration cellulaire de p53, ainsi que son activité, sont contrôlées par I'oncogène $\mathrm{Mdm} 2$, dont l'expression est normalement répartie entre le nucléoplasme et le cytoplasme. Mdm2 se lie à p53, bloquant ainsi son activité de facteur de transcription, et entraînant sa dégradation en facilitant son exportation du nucléoplasme vers le cytoplasme [37-39].

En réponse à des signaux oncogéniques ainsi qu'à la sénescence réplicative, la protéine oncogène ARF stabilise la protéine p53 de la manière suivante: ARF est exclusivement localisée dans le nucléole, et y stabilise p53 en séquestrant $\mathrm{Mdm} 2$, abolissant ainsi le transport cytoplasmique de p53 et la dégradation qui s'ensuit $[40,41]$. La surexpression de ARF entraîne la relocalisation de $\mathrm{Mdm} 2$ dans le nucléole. Une mutation dans la séquence du signal de localisation nucléolaire de ARF, n'affectant pas sa liaison à $M d m 2$, entraîne cepen- 
dant une déstabilisation permanente de p53. Ces observations démontrent clairement que seule la séquestration spatiale de l'oncogène $\mathrm{Mdm} 2$ dans le nucléole inhibe sa fonction de régulateur du taux de p53 dans le nucléoplasme [40].

\section{Point de contrôle du stade pachytène de la méiose}

Au cours de la prophase de la première division de méïose, les deux paires de chromatides sœurs s'apparient transitoirement, permettant l'apparition de recombinaisons. La formation correcte du complexe synaptosomal entre les deux chromosomes-frères, ainsi que le déroulement correct des événements de recombinaison, sont soumis au «point de contrôle du stade pachytène » capable de bloquer le déroulement de la méîse. La protéine Pch2, élément essentiel du contrôle du stade pachytène chez la levure Saccharomyces cerevisiae, est localisée majoritairement dans le nucléole, et n'est exprimée qu'au cours de la méïose [42]. Or, la localisation nucléolaire de la Pch $2 p$ est due à son interaction avec la protéine Sir2. Ainsi, l'abolition de l'expression de Sir2 entraîne la délocalisation de Pch2p de nouveau dans la totalité du nucléoplasme, et aboutit à une perte du contrôle du stade pachytène.

Les protéines Sir2, Net1 et Cdc14 font partie d'un complexe protéique nucléolaire multifonctionnel appelé RENT (regulator of nucleolar silencing and telophase exit) (pour plus de détails, voir [43]).

\section{Conclusions}

Les travaux décrits dans cette synthèse soulignent l'émergence d'une nouvelle vision du nucléole, désormais envisagé comme un organite «plurifonctionnel » [27], impliquée dans une grande variété de fonctions cellulaires. II est clair que la fonction majeure du nucléole demeure la biogenèse des ribosomes. Cependant, il est également responsable de la synthèse d'autres composants de la machinerie d'expression génique. Le nucléole est impliqué dans les modifications post-transcriptionnelles, la maturation nucléolytique, I'assemblage et I'export de différentes familles de petits ARN stables comme les snARN de la machinerie d'épissage, les ARNt, I'ARN télomérase ainsi que l'ARN de la particule SRP, tous issus des machineries de transcription des polymérases II et III. En outre, le nucléole est un compartiment important pour la séquestration de facteurs contrôlant le cycle cellulaire. Les futures études structurales et fonctionnelles du nucléole "plurifonctionnel», cette "boîte magique» de la cellule, conduiront certainement à de nouvelles surprises concernant la biogenèse des ARN cellulaires et le contrôle des mécanismes cellulaires

\section{Remerciements}

Nous remercions Michèle Caizergues-Ferrer, Claire Dugast et Michel Darzacq pour leur relecture critique de ce manuscrit.

\section{RÉFÉRENCES}

1. Wagner R. Einige Bemerkungen und Fragen über das Keimbläschen (vesicula germinativa). Müller's Archiv Anat Physiol Wissenschaft M ed 1835; 373-7.

2. Montgomery TH. Comparative cytological studies, with special regard to the morphology of the nucleolus. J M orphol 1898; $15: 265-582$.

3. Phair RD, Misteli T. High mobility of proteins in the mammalian cell nucleus. Nature 2000; 404: 604-9.

4. Venema I, Tollervey D. Ribosome synthesis in Saccharomyces cerevisiae. Annu Rev Genet 1999; 33: 261-311.

5. Limbach PA, Crain PF, McCloskey JA. Summary: the modified nucleotides of RNA. Nucleic Acids R es 1994; 22 : 2183-96.

6. Agris PF. The importance of being modified: roles of modified nucleosides and $\mathrm{Mg}^{+}$in RNA structure and function. Prog $\mathrm{Nudl} A$ cid Res M ol Biol 1996; 53 : 79-129.

7. Davis FF, Allan FW. Ribonucleic acids from yeast which contain a fifth nucleotide. J Biol Chem 1957; 227: 907-15.

8. Balakin AG, Smith L, Fournier MJ. The RNA world of the nucleolus: two major families of small RNAs defined by different box elements with related functions. Cell 1996; 86: 823-34.

9. Ganot $P$, Caizergues-Ferrer $M$, Kiss $T$. The family of box ACA small nucleolar RNAs is defined by an evolutionarily conserved secondary structure and ubiquitous sequence elements essential for RNA accumulation. Genes Dev 1997; 11: 941-56.
10. Tollervey D, Kiss T. Function and synthesis of small nucleolar RNAs. Curr Opin Cell Biol 1997; 9: 337-42.

11. Kiss-László Z, Henry Y, Bachellerie JP, Caizergues-Ferrer $M$, Kiss T. Site-specific ribose methylation of preribosomal RNA : a novel function for small nucleolar RNAs. Cell 1996; 85 : 1077-88.

12. Cavaillé J, Nicoloso M, Bachellerie JP. Targeted ribose methylation of RNA in vivo directed by tailored antisense RNA guides. Nature 1996; 383: 732-5.

13. Kiss-László $Z$, H enry Y, Kiss T. Sequence and structural elements of methylation guide snoRNA essential for site-specific ribose methylation of pre-rRNA. EMBO J 1998; 17: 797-807.

14. Watkins NJ, Segault V, Charpentier B, et al. A common core RNP structure shared between the small nucleolar box C/ D RNPS and the spliceosomal U4 snRNP. Cell 2000; 103: $457-66$.

15. Tollervey $D$, Lehtonen $H$, Jansen $R$, Kern H, Hurt ÉC. Temperature-sensitive mutations demonstrate roles for yeast fibrillarin in pre-rRNA processing, pre-rRNA methylation, and ribosome assembly. Cell 1993; 72: 443-57.

16. Niewmierzycka A, Clarke S. S-Adenosylmethionine-dependent Methylation in Saccaromyces cerevisiae. J Biol Chem 1999; 274: 814-24.

17. Ganot P, Bortolin ML, Kiss T. Site-specific pseudo-uridine formation in preribosomal RNA is guided by small nucleolar RNAs. Cell 1997; 89: 799-809.

18. $\mathrm{Ni} J$, Tien $A L$, Fournier MJ. Small nucleolar RNAs direct site-specific synthesis of pseudo-uridine in ribosomal RNA. Cell 1997; 89: 565-73.

19. Lafontaine DLH, Bousquet-Antonelli $C$, Henry $Y$, Caizerques-Ferrer M, Tollervey D. The box $\mathrm{H}+A C A$ snoRNAs carry Cbf5p, the putative pseudo-uridine synthase. Genes Dev 1998; 12: 527-37.

20. Zebarjadian $Y$, King T, Fournier MJ, Clarke $L$, Carbon J.' Point mutations in yeast CBF5 can abolish in vivo pseudo-uridylation of rRNA. M ol Biol Cell 1999; 19: 7461-72.

21. Ganot $P$, Jády $B E$, Bortolin $M L$, Darzacq $X$, Kiss T. Nucleolar factors direct the 2'-Oribose methylation and pseudo-uridylation of U 6 spliceosomal RNA. M ol Cell Biol 1999; 19: 6906-17.

22. Tycowski K, You ZH, Graham PJ, Steitz JA. Modification of $\cup 6$ spliceosomal RNA is guided by other small RNAs. M ol Cell 1998; 2: 629-38

23. Jády $B E$, Kiss $T$. A small nucleolar guide RNA functions both in 2'-O-ribose methylation and pseudo-uridylation of the U5 spliceosomal RNA. EM BO 」 2001; 20 : 541-51.

24. Lange TS, Gerbi SA. Transient nucleolar localization of U 6 small nuclear RNA in Xenopus laevis oocytes. M ol Biol Cell 2000; 11 : 2419-28. 


\section{RÉFÉRENCES}

25. Jády BE, Kiss T. Characterisation of the U 83 and U'84 small nucleolar RNAs: two novel 2'-O-ribose methylation guide RNAs that lack complementarities to ribosomal RNAs. Nucleic Acids Res $2000 ; 28$ : 1348-54.

26. Cavaillé J, Bulting $K$, Kiefmann $M$, et al. Identification of brain-specific and imprinted small nucleolar RNA genes exhibiting an unusual genomic organization. Proc $\mathrm{Natl}$ Acad Sci USA 2001; 97 : 14311-6.

27. Pederson $T$. The plurifunctional nucleolus. Nuclèc Acids Res 1998; 26: 3871-6.

28. Politz JC, Yarovoi S, Kilroy SM, Gowda K, Zwieb C, Pederson T. Signal recognition particle components in the nucleolus. Proc Natl A cad Sci USA 2000; 97 : 55-60.

29. Bertand $E, H$ ouser-Scott $F$, Kendall $A$, Singer RH, Engelke DR. Nucleolar localisation of early tRNA processing. Genes Dev 1998; 12: 2463-8.

30. Mitchell JR, Cheng J, Collins K. A box $\mathrm{H}$ / ACA small nucleolar RNA-like domain at the human telomerase RNA 3' end. M ol Cell Biol 1999; 19: 567-76.

31. Narayanan $A$, Lukowiak $A$, Jády $B$, et al. Nucleolar localization signal of Box H/ ACA small nucleolar RNAs. EMBO J 1999; 18: 5120-30.

32. Mitchell JR, Wood E, Collins K. A telomerase component is defective in the human disease dyskeratosis congenita. Nature 1999; 402 : 551-5.

33. Visintin $R$, Craig $K, H$ wang $E S$, Prinz $S$, Tyers $M$, Amon A. The phosphatase Cdc14 triggers mitotic exit by reversal of $\mathrm{Cdk}$ dependent phosphorylation. M ol Cell 1998; 2: 709-18.

34. Jaspersen SL, Charles JF, Morgan DO. Inhibitory phosphorylation of the APC regulator Hctl is controlled by the kinase Cdc28 and the phosphatase Cdc14. Curr Biol 1999; 9 : 227-36.

35. Shou W, Seol JH, Shevchenko A, et al. Exit from mitosis is triggered by Tem1dependent release of the protein phosphatase Cdc14 from nucleolar RENT complex. Cell 1999; $97:$ 233-44.
36. Visintin R, H wang ES, Amon A. Cfil prevents premature exit from mitosis by anchoring Cdc14 phosphatase in the nucleolus. Nature 1999 ; 398: 818-23.

37. Momand J, Zambetti GP, OIson DC, George D, Levine AJ. The mdm-2 oncogene product forms a complex with the p53 protein and inhibits p53-mediated transactivation. Cell 1992; 69: 1237-45.

38. Roth J, Dobbelstein M, Freedman DA, Shenk T, Levine AJ. Nucleo-cytoplasmic shuttling of the hdm2 oncoprotein regulates the levels of the $\mathrm{p} 53$ protein via a pathway used by the human immunodeficiency virus rev protein. EM BO | 1998; 17: 554-64.

39. Freedman DA, Levine AJ. Nuclear export is required for degratation of endogenous $\mathrm{p} 53$ by MDM 2 and human papillomavirus E6. Mol Cell Biol 1998; 18: 7288-93.

40. Weber DJ, Taylor LJ, Roussel MF, Sherr CJ, Bar-sagi D. Nucleolar Arf sequesters Mdm2 and activates p53. Nat Cell Biol 1999; 1: 20-5.

41. Tao W, Levine AJ. p19ARF stabilizes p53 by blocking nucleo cytoplasmic shuttling of Mdm2. Proc Natl Acad Sci USA 1999; 96 : 6937-41

42. San-Segundo PA, Roeder GS. Pch2 links chromatin silencing to meiotic checkpoint control. Cell 1999; 97: 313-24.

43. Cockell MM, Gasser SM. The nucleolus: nucleolar space for RENT. Curr Biol 1999; $9: 575-6$.

\section{TIRÉS À PART}

\section{T. Kiss.}

\section{Summary}

Nucleolus: an «old » cellular organelle with new functions

The nucleolus was the first subnuclear structure described by early microscopists, and has long been considered as a highly specialised organelle, fully dedicated to the biogenesis of cytoplasmic ribosomes. However, over the past few years, a spate of studies has implicated the nucleolus in various cellular processes, including biogenesis of small stable RNAs and cell-cycle regulation. The nucleolar biogenesis of ribosomes includes site-specific synthesis of a large number of 2'-0-methylated nucleotides and pseudouridines in the $18 \mathrm{~S}, 5.8 \mathrm{~S}$ and $28 \mathrm{~S}$ rRNAs. Both 2'-0-methylation and pseudouridylation of rRNAs is guided by small nucleolar RNAs (snoRNAs), which select the correct target nucleotides through formation of transient base-pairing interactions with rRNA sequences. Small nucleolar RNAs have been shown to function in the modification of spliceosomal small nuclear RNAs (snRNAs), and probably of several other celIular RNAs. Other studies strongly support the idea that the nucleolus also contributes to the biogenesis and/ or trafficking of several small stable cellular RNAs. Even more surprisingly, it has recently been demonstrated that spatial confinement of important cell-cycle regulatory proteins in the nucleolus actively contributes to the control of cellular survival and proliferation. 